\title{
Experimental Analysis of Voice and Data Transmission Parameters for Mobile Terminals in Cellular Data Networks
}

\author{
Montserrat Jiménez-Licea, Sergio Vidal-Beltrán, José Luis López-Bonilla \\ Maestría en Ciencias en Ingeniería de Telecomunicaciones, Instituto Politécnico Nacional, Escuela Superior de Ingeniería Mecánica \\ y Eléctrica, México City, México. \\ Email: svidalb@ipn.mx
}

Received November $8^{\text {th }}$, 2010; revised December $26^{\text {th }}, 2010$; accepted December $29^{\text {th }}, 2010$.

\begin{abstract}
This work presents the experimental results related to performance measurements in GSM and GPRS cellular data networks. Two Mobile Terminals were used, in order to take place the scenario. Power Level, Power vs Time, Modulation Analysis, Modulation Spectrum, (BER) Bit Error Rate and Throughput were considered in this analysis.
\end{abstract}

Keywords: GSM, GPRS, Cellular Networks

\section{Introduction}

WCDMA (Wideband Code Division Multiple Access) is a technology of third generation 3G that increases data transmission rates by broadening the signal at the air interface through the Multiple Access Code Division Multiplexing instead of Time Division used by GSM systems. WCDMA supports multimedia services with very high speed and full-motion video, Internet access and videoconferencing. It also easily handles applications that make use intensively of bandwidth such as data and image transmission via Internet. In a communication system based on multiple access, an amount of users want to access the channel simultaneously. To implement a multiple access communication there are several techniques that allow users to share the channel. In the technique of multiple access code division -CDMA-, users can access the channel simultaneously and share the same bandwidth.

WCDMA is a spread spectrum technology [1], which expands the signals over a bandwidth of $5 \mathrm{MHz}$ and is capable of carrying voice and data at the same time. These features allow a data rate of $384 \mathrm{kbps}$ in Release 5 , and a transfer rate in the downlink of up to 2 Mbps in the Release 6, which includes HSPA, and average processing speeds (for file downloads) of 220-320 Kbps [2].

\footnotetext{
*The authors thank the IPN for support provided to this research.
}

Spread spectrum is a technique used to transmit data on a bandwidth considerably larger. Its basic foundation is the "widening" of the transmitted signal over a very wide frequency band, much wider than the minimum bandwidth required transmitting the information that you want to send. This higher bandwidth can be obtained by encoding information with a pseudorandom signal. The coded information is transmitted in the frequency that works for which the sender uses a much higher bandwidth than no encryption is used (direct sequence). The spreading operation is the multiplication of each bit of user data with a sequence of 8 bits of code, called chips $[3,4]$. Currently, WCDMA is the wireless access technology broadest deployed worldwide. WCDMA is a wide band system where the transmitted signal with a rate $\mathrm{R}$ is spread by combining it with a wideband spreading signal, creating a spread signal with a bandwidth $\mathrm{W}$. The effective bandwidth for a WCDMA air interface is $3.84 \mathrm{MHz}$ and with guard bands, as a result the required bandwidth is 5 $\mathrm{MHz}$ [4]. The WCDMA system uses several codes; when the signal is transmitted from the Base Station carries a unique code called Scrambling Code SC, used in the downlink direction for cell/sector separation. This SC is also employed in the uplink direction to separate every user from each other.

Cellular networks were created to transmit voice calls, but currently, it is able to carry multimedia ser- 
vices like data and video. In the following section, the cellular communication generations are described.

In the other hand, the mobile terminal is a critical issue inside of a Cellular Communication System, due to its characteristics of transmission and reception; they defined the quality in the data and voice transmission. This work carried out several experiments mainly focused on the following parameters:

- Power Measurements

- Data transmission measurements

- BER and BLER Tests

GSM, GPRS and WCDMA were the technologies selected in order to deploy the performance analysis. This kind of work cab be used for manufacturers, service providers and user in order to verify the capacity and coverage of cellular data networks.

\section{Performance Parameters in Mobile Terminals in GSM Systems}

The mobile terminal is a critical issue inside of a Cellular Communication System, due to its characteristics of transmission and reception; they defined the quality in the data and voice transmission. In this work, some experiments of the most important parameters in Mobile terminal were carried out. Figure 1 shows the scenery that is necessary to set up to be able to get the experimental results. [2-3]

\subsection{Experimental Scenario}

The elements that are necessary to figure out the experimental scenery are:

- MT8820A Radiocommunications Analyzer, it has a range of frequencies between $30 \mathrm{MHz}$ to $2.7 \mathrm{GHz}$, The range result enough to get the experimental measures into the cell phones. This equipment allowed getting measurements of Transmission/Reception in technologies like: W-CDMA/HSDPA, GSM/GPRS/EGPRS, cdma 2000, $1 \mathrm{x}$ (IS-2000), cdma2000 1xEV-DO, PDC and PHS/ ADVANCED [2-3].

- Shielded Box is used to avoid interferences from the surrounding Base Stations. Such device works between 800 and $2500 \mathrm{MHz}$, and gives $60 \mathrm{~dB}$ of isolation [2-3].

- Coaxial cable is used to connect the MT8820A to

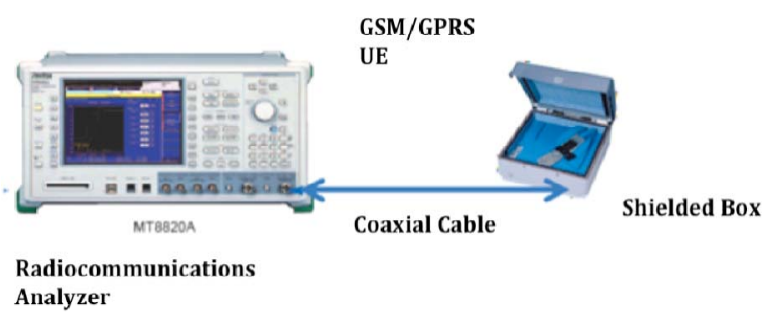

Figure 1. Test Scenario. the Shielded box. Type- $\mathrm{N}$ connectors are used

- Two mobile terminals that operate in GSM, as much as WCDMA; they are iPhone 3G and Nokia 6210.

A USIM card is installed for the test. It was necessary to register the USIM with the Radiocommunications analyzer.

\subsection{Power Measurements in Voice Calls}

Tests were made in the Radio-communications Laboratory of the Master Degree in Telecommunications Engineering (IPN). Once the equipments were properly installed, the following procedure took place. [4]

- The Radio-communications analyzer was powered up, and the technology parameters were configured, according to GSM, GPRS, EDGE or WCDMA technology.

- Voice transmission parameters were configured, in order to establish a voice call.

- The UE is powered up and was placed into the shielded box. The shielded box allows to focus on the power inside the box and avoiding electromagnetic interferences from the surrounding cellular antennas

- The MTT8820A is configured with a power between $-40 \mathrm{dBm}$ to $-50 \mathrm{dBm}$

- UE establish communications with the Analyzer and the state Registered have to appear in the MT8820A's screen.

- The power level was slowly decremented until the Analyzer detects errors

The Power levels are registered, as much as the errors that the Analyzer captured.

\subsection{Data Transmission Measurements}

Once the equipment, described in the previous section, was properly installed, the following procedure took place.

- The Radiocommunications analyzer was powered up, and the technology parameters were configured, according to GSM, GPRS, EDGE or WCDMA technology.

- Voice transmission parameters were configured, in order to establish a voice call.

- The UE is powered up and was placed into the shielded box. The shielded box allows to focus on the power inside the box and avoiding electromagnetic interferences from the surrounding cellular antennas

- The MTT8820A is configured with a power between $-40 \mathrm{dBm}$ to $-50 \mathrm{dBm}$

- UE establish communications with the Analyzer and the state Registered have to appear in the MT 8820A's screen.

- A data Server (http) is connected into the same network segment that the MT8820A, and the connectivity between them is verified

- The UE makes a data request in order that to down- 
load a file with a size previously defined

- The download times are registered.

\section{Power Measurements in Voice Calls for GSM}

Figure 2 shows the parameters set up of the MT8820A for GSM. [5]

In the Figure 2, the first section shows that GSM is configured, in the part 2, the uplink and downlink frequencies are established; the power in the base station is specified in the Section $3(-40 \mathrm{dBm})$, the power in the mobile station $(33 \mathrm{dBm})$ and it is used the control of power, the last in the part 4 shows the power in the base station that it is received for the mobile station (11.13 dBm). Initially, the measurement was made on the Nokia 6120, those were: Power Level, Power vs Time, Modulation Analysis, Modulation Spectrum, (BER) Bit Error Rate, the performance in the mobile terminal shows an acceptable level in the range allowed in the specification, the Figures 3-4 show some results.

In this scenario, it does not appeared errors, because the communication is efficient.

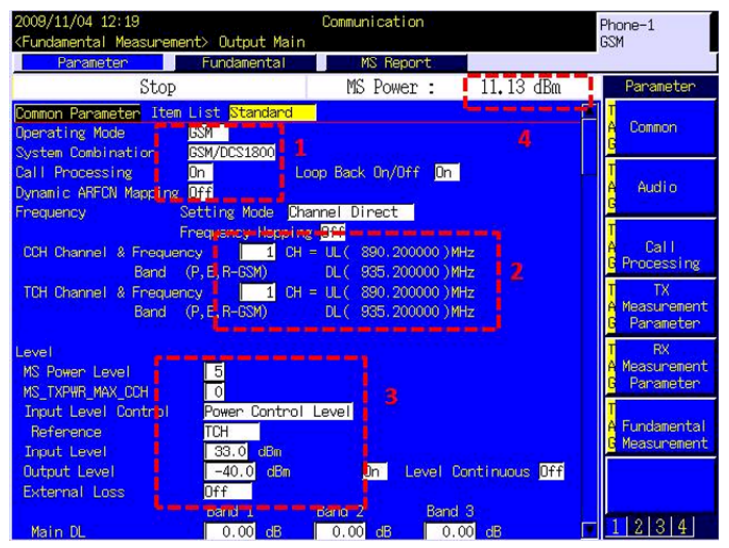

Figure 2. Configuration Parameters in GSM.

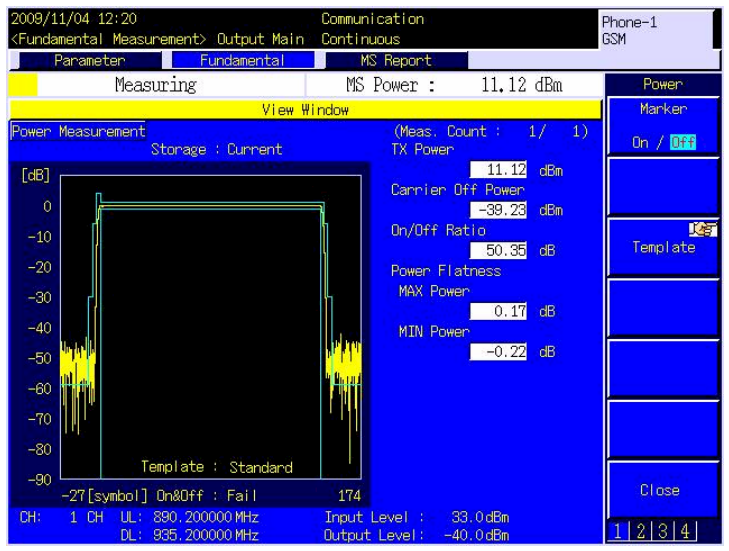

Figure 3. Power measurement.

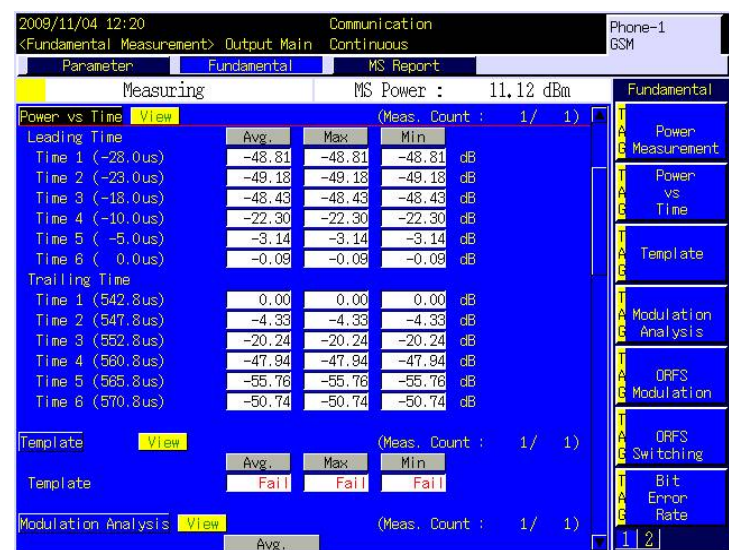

Figure 4. Measurement Power vs Time.

\subsection{Minimum Power Reception in Voice Calls}

The second part of the test consists in decreasing the power level of the base station detecting errors in the transmission; the power in which the Nokia equipment established the communication without errors is $-86 \mathrm{dBm}$.

The same test was applied to the mobile iPhone, the power where error came up were $-80 \mathrm{dBm}$. The Figures 5-6 show the results got for this mobile.

It should be pointed out that Nokia 6120 as iPhone initially satisfy the measurements parameters. As much as the power level is decreased differences between them appear. It should be pointed out that initially the Nokia 6120 as iPhone satisfy the measurements parameters.

Once the power level is decreased, we can notice that the Nokia 6120 has better reception sensitivity; the errors in the transmission are detected until $-86 \mathrm{dBm}$, and the minimum power level without errors for the iPhone is $-80 \mathrm{dBm}$. This difference $(6 \mathrm{dBm})$ is a big deal; due to the distance that the Nokia can establish a voice call is greater than iPhone.

\section{GPRS Tests Measurements}

When the UE is able to support GPRS, external data transmission parameters can be characterized. These measurements just can take place when GPRS and WCDMA networks are considered [4]. The configurations parameters are shown in Figure 7. We can appreciate in Figure 7, that GPRS system is configured; the power level of the base station $(-40 \mathrm{dBm})$, the power of the mobile terminal (33 $\mathrm{dBm}$ ), just like the power in the channels likewise establishes the power control.

The Base Station receives $7.89 \mathrm{dBm}$ of power of the mobile terminal. It is possible to established different types of tests (Test Mode A, Test Mode B, BLER y External Packet). In this work is just used mode BLER to prove the capacity of reception in the mobile terminal, to 


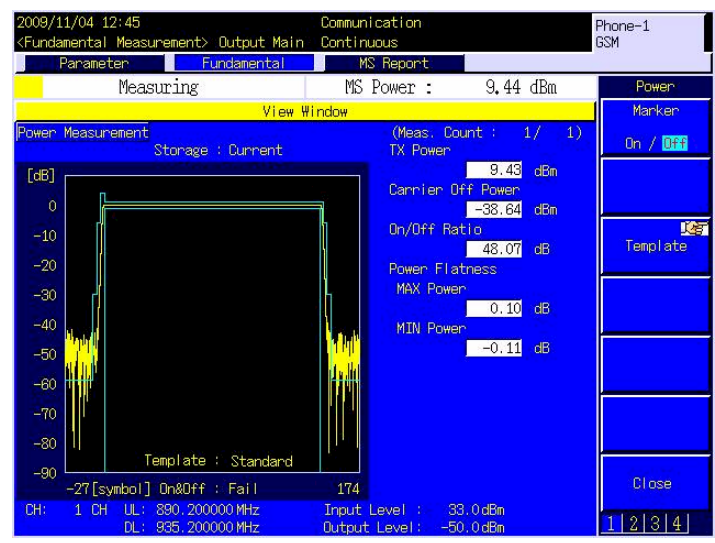

Figure 5. Power measurement.

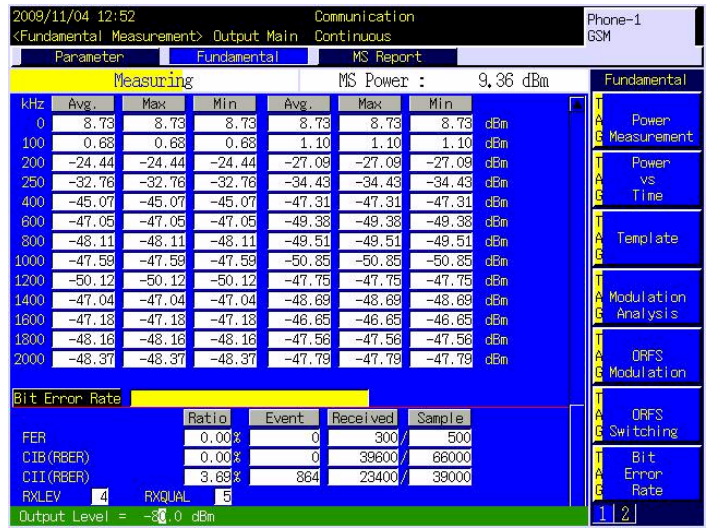

Figure 6. Measurements of BER.

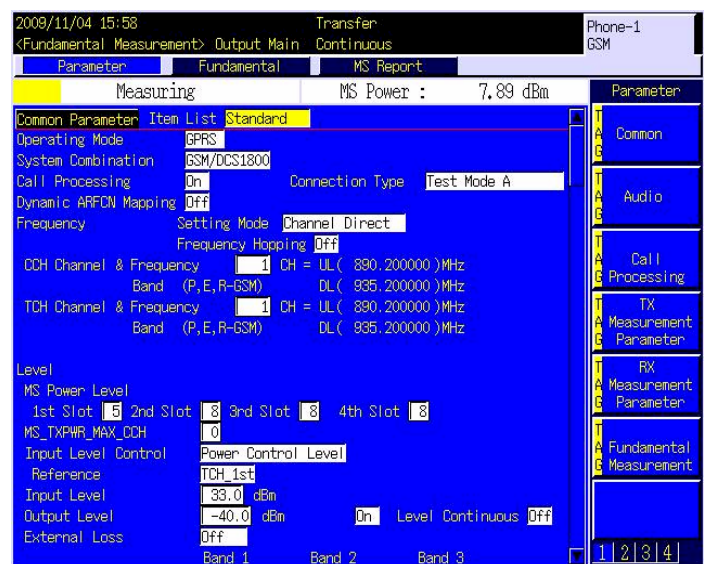

Figure 7. Power measurement.

a measurement of power of the base station decreases. The Figure 8, shows the power in which the mobile station starts to present errors.

GPRS contains different modulation schemes, to compensate the errors in the transmission in respect to the distance increase. [6,7]. The modulation scheme that it is used first is CS-4, it has the highest bit rate transmis-

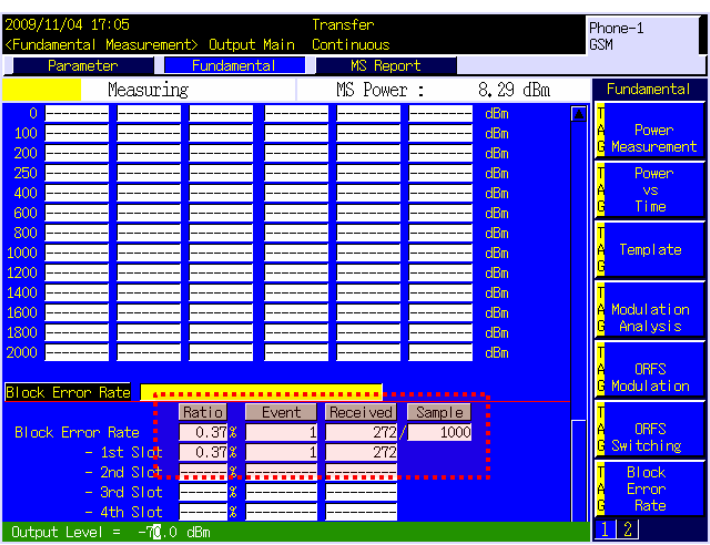

Figure 8. BLER Measurements.

sion of data, that scheme is used when the base station is closer to the mobile device. The modulation scheme CS-1 is the most robust, because it has more bit of redundancy, but it presents the lowest bit rate transmission. [6,7]. When the station mobile works with CS-1, the terminal presents errors in $-76 \mathrm{dBm}$. The Table 1 shows a resume with the obtained results for Nokia and iPhone UEs.

Only the Coding schemes CS1 and CS4 were used, in order to consider the best and the worst scenario.

\subsection{External Data Measurements}

In order to make these measurements the MT8820A, a web server, a shielded box and two UE were used. A Data file was requested for the UE to the server. The MT8820A is used as a base Station and is able to capture all the parameters considered. The following scenario was installed (Figure 9).

The IP network segment 172.168.1.X was used with a network mask with 24 bits. Table 2, shows the IP Addressing scheme used.

Table 1. Experimental Results for GPRS.

\begin{tabular}{ccc}
\hline User Equipment & $\begin{array}{c}\text { Minimum power level with- } \\
\text { out errors }\end{array}$ & $\begin{array}{c}\text { Coding } \\
\text { scheme }\end{array}$ \\
\hline Nokia 6120 & $-70 \mathrm{dBm}$ & CS-4 \\
Nokia 6120 & $-76 \mathrm{dBm}$ & CS-1 \\
iPhone & $-64 \mathrm{dBm}$ & CS-4 \\
iPhone & $-69 \mathrm{dBm}$ & CS-1 \\
\hline
\end{tabular}

Table 2. IP Addressing.

\begin{tabular}{cc}
\hline IP of the Server & 172.168 .1 .183 \\
\hline IP of the MT8820A & 172.168 .1 .101 \\
IP of the UE & 172.168 .1 .99 \\
\hline
\end{tabular}




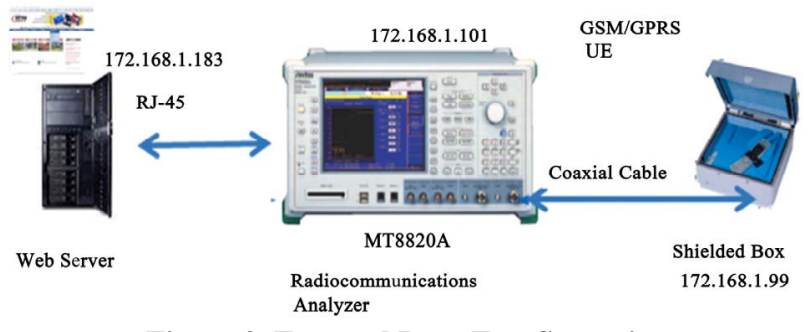

Figure 9. External Data Test Scenario.

The Attached State has to be reached in order to get communication between the MT8820A and the UE. Once the communication is established connectivity test was made. The attached state synchronizes the UE with the network. The Figure 10, shows that the communications at the IP level is establish without problems.

From the above figure, it could be pointed out that the time to get an answer from the UE is greater than the time observed for LAN networks, while the answer for the MT8820A is $3 \mathrm{~ms}$, the UE did it in 1 second. Once the scenario was installed, the data transfer can take place, the data rate can be measured and different coding schemes were used. The size of the file was $200 \mathrm{~KB}$, the experimental results are shown in the Table 3 . As is showed in the table below, there were differences between the theoretical data and the measured data transferred. This fact can be an effect of the signal delay when travels from the MT8820A to the UE. It should be remembered that the test was made using just one channel. GPRS has 4 channels available. Additionally, it has to be considered that in data communications, the OSI layered protocol is used; and each layer, aggregates information to the original data.

\section{WCDMA Measurements}

For the WCDMA measurements, the MT8820A were configured as follows (Figure 11);

For the testing of WCDMA, the band V of the UMTS channels is selected [5], corresponding to Mexico; the channels 1087 (887.5 MHz) and 862 (842.5 MHz) are used respectively for downlink and uplink. We used a

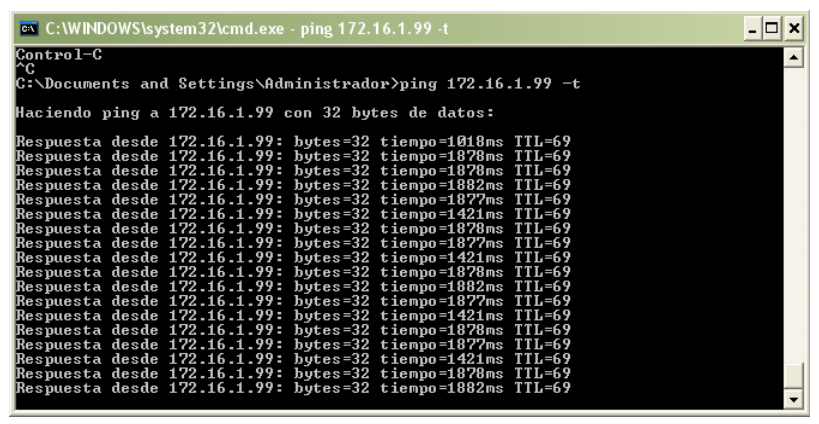

Figure 10. Connectivity test (Ping).
Table 3. Experimental data rates for GPRS.

\begin{tabular}{|c|c|c|c|c|c|}
\hline 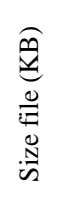 & 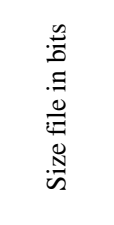 & 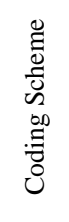 & 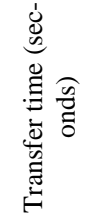 & 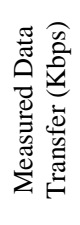 & 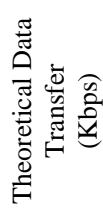 \\
\hline 200 & 1600000 & CS1 & 240.00 & 6.67 & 8000 \\
\hline 200 & 1600000 & CS2 & 167.33 & 9.56 & 12000 \\
\hline 200 & 1600000 & CS3 & 143.11 & 11.18 & 14400 \\
\hline 200 & 1600000 & CS4 & 110.00 & 14.55 & 20000 \\
\hline
\end{tabular}

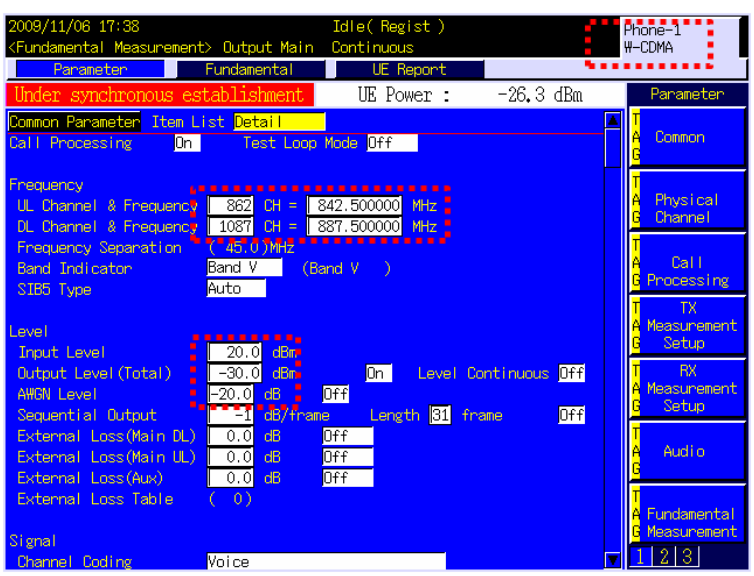

Figure 11. MT8820A configuration for WCDMA.

power level of $-30 \mathrm{dBm}$ for the transmission of $\mathrm{MT}$ 8820A.

\subsection{BER/BLER Measurements}

BER test mode is configured in the MT8820A and it proceeded to decrease the power level until we observe errors in the Analyzer. The Table 4 and Figure 12 show the results of the two terminals under evaluation.

\subsection{External Data Measurements}

As with GPRS and EDGE, WCDMA technology allows data transmission tests from an external server to the mobile terminal via PPP and IP connections. Radio Analyzer (MT8820A) enables communication between a server and a client using the air interface of the mobile terminal. The scenario is shown in Figure 9. For this test, the following parameters were configured in the MT8820A [4].

$\checkmark$ The IP Default Gateway field is set to 0.0.0.0

$\checkmark$ Call Processing parameter must be enabled,

$\checkmark$ The Test Loop Mode must be disabled and

$\checkmark$ The Channel coding must be Packet, to indicate that we establish a data session.

$\checkmark$ The IP addressing is shown in Figure 9. 
Table 4. BER/BLER results.

\begin{tabular}{ccc}
\hline User Equipment & $\begin{array}{c}\text { Power level } \\
\text { without errors }\end{array}$ & $\begin{array}{c}\text { Power level with } \\
\text { errors }\end{array}$ \\
\hline Nokia 6120 & $-80 \mathrm{dBm}$ & $-82 \mathrm{dBm}$ \\
iPhone & $-74 \mathrm{dBm}$ & $-75 \mathrm{dBm}$ \\
\hline
\end{tabular}

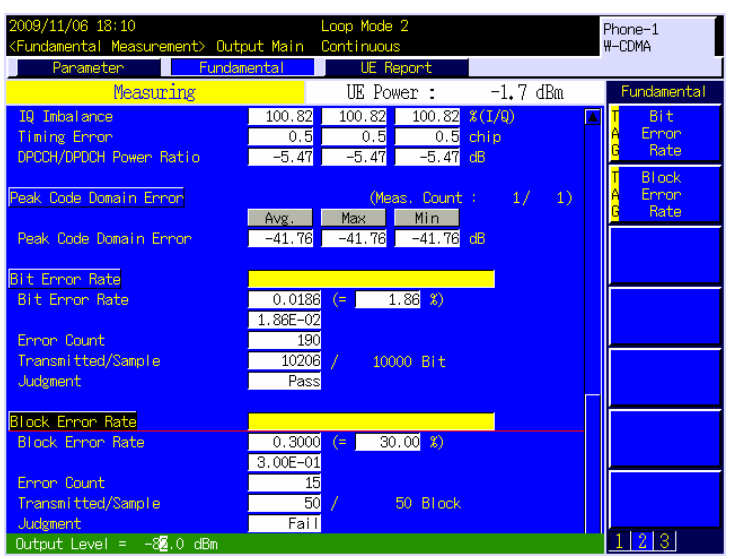

Figure 12. BER/BLER results for Nokia 6120.

Subsequently, we introduce the mobile terminal within the shielded box and the Analyzer proceeds to Register the equipment under test, and once this is done, the Communication mode is set indicating that the equipments are linked and data transfer can take place. In this case, due to the characteristics of the measuring equipment, we can only test the downlink 384 Kbps theoretical speed [6,7]. The power level configuration of the base station and mobile terminal was carried out according to what is specified in Figure 11. Once that is done all the configuration and connectivity testing, we proceed to the download of information from a local web site to test the transmission rates using different coding schemes. The user equipment downloaded a file (200 KB) from a local web server (Figure 9). Table 5 shows the results of the test.

As we can see, there is a difference between the data rate measured and data rate theoretical provided by the WCDMA downlink. As with GPRS, the information added by the IP transmission, as well the WCDMA air interface caused an increase in the amount of information transmitted in the communication, which influence the transmission rate measured.

\section{Conclusions}

In this paper is shown the experimental results done to the two mobile terminals in GSM, GPRS and WCDMA
Table 5. Data rate for WCDMA.

\begin{tabular}{ccccc}
\hline $\begin{array}{c}\text { Tamaño } \\
\text { de archivo } \\
(\text { KB })\end{array}$ & $\begin{array}{c}\text { Tamaño de } \\
\text { Archivo } \\
\text { (bits) }\end{array}$ & $\begin{array}{c}\text { Tiempo de } \\
\text { Transferencia } \\
(\text { seg })\end{array}$ & $\begin{array}{c}\text { Tasa de } \\
\text { Transmisión } \\
\text { Medida (Kbps) }\end{array}$ & $\begin{array}{c}\text { Tasa de } \\
\text { Transmisión } \\
\text { Teórica } \\
\text { (Kbps) }\end{array}$ \\
\hline 200 & 1600000 & 5.67 & 292.68 & 384 \\
\hline
\end{tabular}

systems. It was verified in the beginning that the terminals have the required specifications. Later they were measure in which the terminals started to present errors, and it results indicators of the ratio of the cells. For the transmission of data, it was found the power limits changing the modulation schemes. The limit power is an important data for designing and planning of a radio network. This task established the tests for a deep analysis from the point of view of coverage in capacity of radio. In future tasks analysis of capacity in transmission will be presented depending on the distance, slots and modulation schemes. It should be remarked that this kind of research is important, due to their results has a great impact at different levels; like that planning, research, manufacturing, scientific and academic areas.

\section{REFERENCES}

[1] H. Holma and A. Toskala, "WCDMA for UMTS-HSPA Evolution and LTE,” 4th Edition, John Wiley \& Sons, Chichester, 2007.

[2] Practical Tips on WCDMA Measurements, Application Note No. 11410-00378, Revision B Printed in United States 2008-09.

[3] R. Blake, "Sistemas Electrónicos de Comunicaciones, Thomson," 2nd Edition, Addison-Wesley, New York, 2004.

[4] Application Note No 11410-00378 Revision B, "Practical Tips on WCDMA Measurements,” Anritsu, 2009. http:// www.us.anritsu.com

[5] K. Tachikawa, "WCDMA Mobile Communications System,” John Wiley \& Sons, Chichester, 2002.

[6] H. Holma and A. Toskala, "WCDMA for UMTS-Radio Access for Third Generation Mobile Communications," John Wiley \& Sons, Chichester, 2004.

[7] C. Chevallier, C. Brunner, A. Garavaglia, K. P. Murray and K. R. Baker, "WCDMA Deployment Handbook Planning and Optimization Aspects,” John Wiley \& Sons, Chichester, 2006. doi:10.1002/0470035749 